\title{
A CHARACTERISTIC ZERO NON-NOETHERIAN FACTORIAL RING OF DIMENSION THREE( $\left.{ }^{1}\right)$
}

BY

\author{
JOHN DAVID
}

ABSTRACT. This paper shows the previously unknown existence of a finite dimensional non-Noetherian factorial ring in characteristic zero. The example, called "J", contains a field of characteristic zero and is contained in a pure transcendental extension of degree three of that field. $J$ is seen to be an ascending union of polynomial rings and degree functions are introduced on each of the polynomial rings. These are the basic facts that enable it to be seen that two extensions of $J$ are Krull. One of these extensions is a simple one and the other is a localization of $J$ at a prime ideal $P$. In the case of the latter extension, it is necessary to show that the intersection of the powers of $P$ is zero. As $J$ is the intersection of these two extensions, a theorem of Nagata is all that is needed to show then that $J$ is factorial. It is easily proved that $J$ is non-Noetherian once it is known to be factorial.

0 . Notation and introduction.

(a) Notation. (i) $k$ is any field of characteristic zero.

over $k$.

(ii) $x, y, a_{0}, \alpha_{1}, \cdots, a_{k}, \cdots$ are algebraically independent variables

(iii) $s$ is the set map from $\mathrm{Z}^{+}$to $\mathrm{Z}^{+}$given by

$s(1)=2, \quad s(2)=3, \quad s(n)=s(n-2) \cdot \ldots \cdot s(2) s(1) \cdot n, \quad n \geq 3$.

(iv) For $N=2,3, \cdots, I_{N}^{\prime}$ is the following ideal in $k\left[x, y, \alpha_{0}, \alpha_{1}, \cdots, \alpha_{N}\right]$ :

$$
\begin{array}{r}
I_{N}^{\prime}=\left(x \alpha_{1}-y, x \alpha_{2}+\alpha_{1}^{s(2)}-\alpha_{0}, x \alpha_{3}+\alpha_{2}^{s(3)}-\alpha_{1}, x \alpha_{4}+\alpha_{3}^{s(4)}-\alpha_{2}, \ldots,\right. \\
\left.x \alpha_{i-1}+\alpha_{i-2}^{s(i-1)}-\alpha_{i-3}, \ldots, x \alpha_{N}+\alpha_{N-1}^{s(N)}-\alpha_{N-2}\right),
\end{array}
$$

$I_{1}^{\prime}=\left(x \alpha_{1}-y\right)$.

Presented to the Society, January 26, 1973; received by the editors July 19, 1972.

AMS (MOS) subject classifications (1970). Primary 13F15, 1.3E05; Secondary 13A15, 13F 20, 13C15, 13 H99.

Key words and phrases. Factorial, Krull dimension, Noetherian, polynomial ring, degree function.

( $\left.{ }^{1}\right)$ This work forms part of the author's doctoral dissertation at the University of Rochester. He wishes to thank Warren May for his kind help.

Copyright $(1973$, American Mathematical Society 
For $N=1,2, \cdots, R_{0}=k\left[x, y, a_{0}\right], R_{N}=k\left[x, y, \alpha_{0}, \cdots, \alpha_{N}\right] / I_{N}^{\prime}$.

(v) For $N=1,2, \cdots$ we define a map

$$
b_{N}: k\left[x, y, a_{0}, a_{1}, \cdots, \alpha_{N}\right] \rightarrow k\left(x, y, \alpha_{0}\right)
$$

as follows:

$$
b_{N} \mid k\left[x, y, a_{0}\right]=\operatorname{id}_{k}\left[x, y, a_{0}\right], \quad b_{N}\left(a_{1}\right)=y / x .
$$

For $i=2,3, \cdots, b_{N}\left(a_{i}\right)=\left(-b_{N}^{s(i)}\left(a_{i-1}\right)+b_{N}\left(a_{i-2}\right)\right) / x$. We now have the following exact diagram:

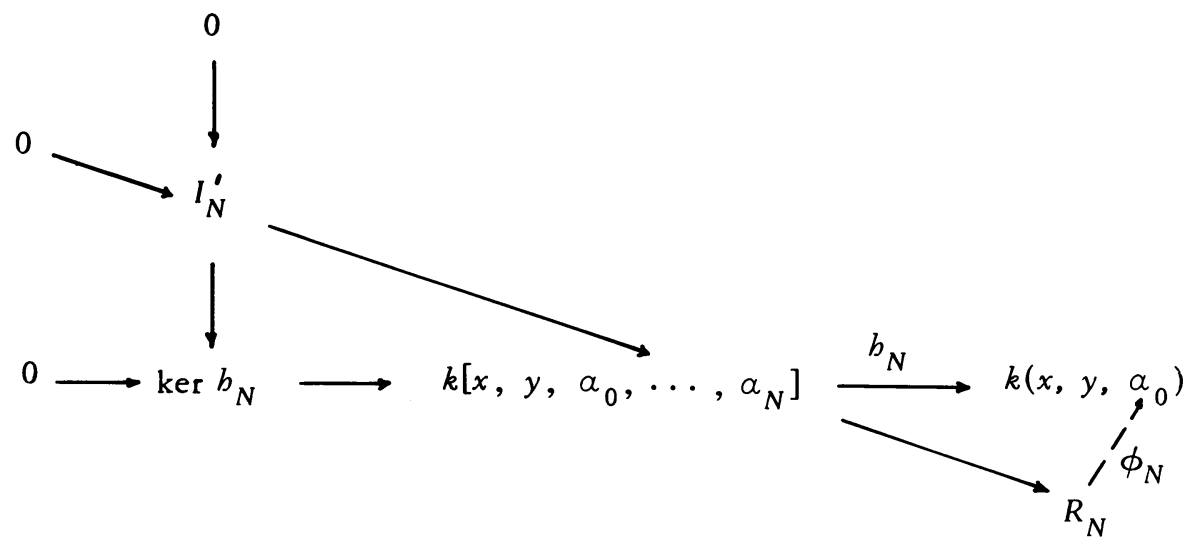

and so we get the map $\phi_{N}=\bar{b}_{N}$.

(vi) For $N=1,2, \cdots$ define

$$
J_{N}=\phi_{N}\left(R_{N}\right), \quad J_{-1}=J_{0}=k\left[x, y, a_{0}\right], \quad J=\bigcup_{N=0}^{\infty} J_{N} .
$$

(b) The major result concerning $\mathrm{J}$.

Theorem 9. $J$ is a non-Noetherian factorial ring of dimension three in characteristic zero.

General method of proof. We first show that $\phi_{N}$ is an isomorphism and $J_{N}$ is a polynomial ring in three variables. We use this isomorphism freely to make the calculations easier. We show that $x$ is a prime element in $J$ and that $J=J(x) \cap$ $J[1 / x]$. Next we show $J[1 / x]$ is Krull; indeed it is a quotient ring of a polynomial ring in three variables. We then complete the major task, to show that $J_{(x)}$ is a rank one discrete valuation ring. We accomplish this by proving $\bigcap(x)^{i}=(0)$. We introduce degree functions on $J_{N}$, called

$$
\begin{array}{lll}
(N) & (N) & (N) \\
\operatorname{deg} & \operatorname{deg}_{\beta_{N}}, & \operatorname{deg}_{\beta_{N-1}}
\end{array}
$$


Note that $J_{N}$ is a polynomial ring in three variables $x, \beta_{N}, \beta_{N-1}$. We look at expansions of an arbitrary element $\alpha$ of $J$ in $J_{N}$ for $N$ large enough and show that if there exists $N$ such that $s(N+1)>\operatorname{deg}_{\beta_{N}}^{(N)} \alpha$ then $\left.x^{(\operatorname{deg}} \underset{x}{(N)}(a)+1\right)$ does not divide $\alpha$ in $J$. Also we show that there does indeed exist such an $N$ for each $\alpha$ of $J$, by looking again at expansions of $\alpha$ in $J_{m}$ for $m$ sufficiently large, and noting the rate of growth of $s$. Consequently we get $\bigcap(x)^{i}=0$. It may be noted here that characteristic zero is essential for these arguments.

That $J$ is Krull follows immediately. That $J$ is three dimensional and nonNoetherian involves some simple combinatorial arguments.

Remark. The author wishes that $J$ be called an "Ericka ring", and that the class of rings into which $J$ logically falls be called the class of "Ericka rings". This class is those rings, commutative domains with identity, derived by adding some variables to a field ringwise and then "dividing out" infinitely often, as in the case of $J$.

(c) Two theorems.

Theorem 1 (Nagata [1, p. 21]). Let $A$ be a Krull ring, $S$ a multiplicatively closed set in $A, 0 \notin S$, generated by prime elements of $A$. Then if $S^{-1} A$ is factorial, so is $A$.

Theorem 2 (Samuel [2, p. 28]). Let $A$ be factorial, $a, b \in A$ with $(a, b)=1$. Let $X$ be a variable over $A$. Then $a X-b$ is prime in $A[X]$.

1. The maps $\phi_{N}$ are coherent injections, $x$ is prime in $J$, and $J$ is a three dimensional domain.

Lemma 1.1. $\bar{x}$ is prime in $R_{i}$ for all $i$.

Proof. It suffices to show that $Q=\left(\alpha_{1}^{s(2)}-\alpha_{0}, \alpha_{2}^{s(3)}-\alpha_{1}, \cdots, \alpha_{N-1}^{s(N)}-\alpha_{N-2}\right)$ is prime in $k\left[a_{0}, a_{1}, \cdots, \alpha_{N-1}\right]$. We will show something stronger:

$$
k\left[\alpha_{0}, \cdots, \alpha_{N-1}\right] / Q \cong k\left[\alpha_{N-1}\right]
$$

in a natural way, and we will use induction on this.

$k\left[\alpha_{0}, \alpha_{1}\right] /\left(\alpha_{1}^{s(2)}-\alpha_{0}\right)$ is a domain, by Eisenstein, and thus

$$
\phi: k\left[\alpha_{0}, \alpha_{1}\right] /\left(\alpha_{1}^{s(2)}-\alpha_{0}\right) \rightarrow k\left[\alpha_{1}\right]
$$

induced by $\alpha_{0} \mapsto \alpha_{1}^{s(2)}, \alpha_{1} \mapsto \alpha_{1}$, and $\mathrm{id}_{k}$ is an isomorphism.

Assume $\phi: k\left[\alpha_{0}, \alpha_{1}, \cdots, \alpha_{m}\right] /\left(\alpha_{1}^{s(2)}-\alpha_{0}, \cdots, \alpha_{m}^{s(m+1)}-\alpha_{m-1}\right) \rightarrow k\left[\alpha_{m}\right]$ induced by

$$
a_{p} \mapsto a_{m}^{\Pi_{i=1}^{m-p} s(i+p+1)}, \quad 0 \leq p \leq m-1
$$

and $\mathrm{id}_{k}\left[a_{m}\right]$ is an isomorphism. Then 


$$
\begin{aligned}
& k\left[a_{0}, \ldots, \alpha_{m+1}\right] /\left(\alpha_{1}^{s(2)}-\alpha_{0}, \cdots, a_{m+1}^{s(m+2)}-\alpha_{m}\right) \\
& \cong_{(1)}\left(k\left[\alpha_{0}, \ldots, \alpha_{m}\right] /\left(\alpha_{1}^{s(2)}-\alpha_{0}, \cdots, \alpha_{m}^{s(m+1)}-\alpha_{m-1}\right)\right)\left[a_{m+1}\right] /\left(\alpha_{m+1}^{s(m+2)}-\bar{\alpha}_{m}\right) \\
& \cong_{(2)} k\left[\alpha_{m}\right]\left[a_{m+1}\right] /\left(\alpha_{m+1}^{s(m+2)}-\alpha_{m}\right) \equiv V_{m+1},
\end{aligned}
$$

where the composition of the two isomorphisms is the map $\phi^{\prime}$ :

$\phi^{\prime}$ is natural, i.e.

$$
\begin{aligned}
& \phi^{\prime}\left(\bar{\alpha}_{p}\right)=\bar{\alpha}_{m}^{\mathbf{n}_{i=1}^{m-p} s(p+i+1)}, \quad 0 \leq p \leq m-1 \\
& \phi^{\prime}\left(\bar{\alpha}_{m}\right)=\bar{\alpha}_{m}, \quad \phi^{\prime}\left(\bar{\alpha}_{m+1}\right)=\bar{\alpha}_{m+1} \quad \text { and }\left.\quad \phi^{\prime}\right|_{k}=\mathrm{id} .
\end{aligned}
$$

By Eisenstein, $V_{m+1}$ is a domain. From dimension theory we have $V_{m+1} \cong(3)$ $k\left[\alpha_{m+1}\right]$ induced by

$$
\alpha_{m} \mapsto a_{m+1}^{s(m+2)}, \quad a_{m+1} \mapsto a_{m+1}, \quad \mathrm{id}_{k} .
$$

Thus the composition of all these isomorphisms (1), (2), (3), gives us the natural isomorphism to satisfy the induction assumption, and to prove the lemma.

Lemma 1.2. $\bar{x} \nmid-\bar{\alpha}_{N-1}^{s(N)}+\bar{\alpha}_{N-2}$ in $R_{N-1}, N \geq 2$.

Proof. Suppose there exist $b_{i}, q \in k\left[x, y, \alpha_{0}, \cdots, \alpha_{N-1}\right]$ such that $\alpha_{N-1}^{s(N)}-$ $a_{N-2}=q x+b_{1}\left(x a_{1}-y\right)+\cdots+b_{N-1}\left(x \alpha_{N-1}+a_{N-2}^{s(N-1)}-a_{N-3}\right)$. Since $s(N-1)>1$, the $a_{N-2}$ term on the left cannot be cancelled and the equation is impossible.

Proposition 1. Let $\beta_{0}=\alpha_{0}, \beta_{1}=y / x, \beta_{2}=\left(-\beta_{1}^{s(2)}+\beta_{0}\right) / x, \beta_{i}=$ $\left(-\beta_{i-1}^{s(i)}+\beta_{i-2}\right) / x$ for $i \geq 3$. Then $\phi_{N}\left(\bar{\alpha}_{i}\right)=\beta_{i}$ and $\phi_{N}: R_{N} \rightarrow J_{N-1}\left[\beta_{N}\right]$ is an isomorphism and $R_{N}$ is factorial of dimension 3. Thus it follows that the $\phi_{N}$ are coberent in the sense that $J_{N} \subseteq J_{N+1}$ and the following diagram commutes:

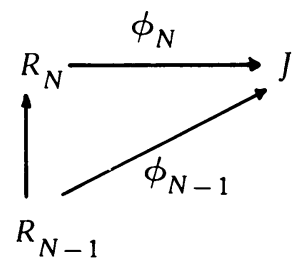

where $R_{N-1} \rightarrow R_{N}$ is the natural map.

Proof. By induction on $N$ :

$N=0$ : Clear.

$N=1: \phi_{1}: k\left[x, y, \alpha_{0}, \alpha_{1}\right] /\left(x \alpha_{1}-y\right) \rightarrow k\left[x, y, \alpha_{0}, y / x\right]=J_{0}\left[\beta_{1}\right]$ is an isomorphism by Theorem 2 .

Since $k\left[x, y, \alpha_{0}, y / x\right]=J_{0}\left[\beta_{1}\right]$ is clearly a polynomial ring in three variables, 
it is factorial of dimension 3. Thus $R_{1}$ is also.

Now we assume $N>1$ and that the proposition is true for $N-1$. First we show that $\phi_{N}: R_{N} \rightarrow J_{N-1}\left[\beta_{N}\right]$ is an isomorphism: We have $\phi_{N-1}: R_{N-1} \rightarrow$ $J_{N-1}$ an isomorphism and $\bar{\phi}_{N-1}: R_{N-1}[X] /\left(\bar{x} X+\bar{\alpha}_{N-1}^{s(N)}-\bar{\alpha}_{N-2}\right) \rightarrow$ $J_{N-1}[X] /\left(x X+\beta_{N-1}^{s(N)}-\beta_{N-2}\right)$ is an isomorphism. Since $R_{N-1}$ is assumed factorial, by Theorem 2 and Lemmas 1.1 and 1.2, l: $R_{N-1}[X] /\left(\bar{x} X+\bar{\alpha}_{N-1}^{s(N)}-\bar{\alpha}_{N-2}\right) \rightarrow$ $R_{N}$ is an isomorphism where

$$
\begin{aligned}
& l\left(\bar{a}_{i}\right)=\bar{\alpha}_{i}, \quad i<N, \quad l l_{k}=\mathrm{id}, \\
& l(\vec{x})=\bar{x}, \quad l(y)=\bar{y}, \quad l(x)=\bar{a}_{N} .
\end{aligned}
$$

Thus the map $\epsilon: R_{N} \rightarrow J_{N-1}[X] /\left(x X+\beta_{N-1}^{s(N)}-\beta_{N-2}\right)$ where $\epsilon=\bar{\phi}_{N-1} \circ l^{-1}$ is an isomorphism. Since by induction $J_{N-1}$ is factorial and $\phi_{N-1}: R_{N-1} \rightarrow J_{N-1}$ is an isomorphism, from Lemmas $1.1,1.2$ and Theorem 2, we can conclude that

$$
\sigma: J_{N-1}[X] /\left(x X+\beta_{N-1}^{s(N)}-\beta_{N-2}\right) \rightarrow J_{N-1}\left[\beta_{N}\right]
$$

is an isomorphism where

$$
\left.\sigma\right|_{J_{N-1}}=\text { id } \quad \text { and } \quad \psi[X]=\beta_{N} \cdot
$$

Therefore $\phi_{N}=\sigma \circ \epsilon: R_{N} \rightarrow J_{N-1}\left[\beta_{N}\right]$ is an isomorphism.

To complete Proposition 2, we must show that $R_{N}$ is a three dimensional factorial ring. To show $R_{N}$ factorial, we observe that

$$
\psi: k\left[x, y, \alpha_{0}, \cdots, \alpha_{N}\right] \rightarrow k\left[x, \alpha_{N-1}, a_{N}\right]
$$

by (inductively)

$$
\begin{gathered}
\psi\left(a_{N-2}\right)=x a_{N}+a_{N-1}^{s(N)} \\
\cdots \\
\psi\left(a_{N-K}\right)=x \psi\left(a_{N-K+2}\right)+\psi\left(a_{N-K+1}^{s(N-K+2)}\right) \\
\cdots \\
\left.\psi\right|_{k\left[x, a_{N}, a_{N-1}\right]}=\mathrm{id}
\end{gathered}
$$

is such that $\operatorname{ker} \psi \supseteq I_{N}^{\prime}$. So we get the following exact sequence

$$
R_{N} \stackrel{\bar{\psi}}{\rightarrow} k\left[x, \alpha_{N-1}, \alpha_{N}\right] \rightarrow 0 .
$$

As shown above, $R_{N}$ is a domain; if we can now show it to have dimension less than or equal to three, then $\bar{\psi}$ will be an isomorphism and $R_{N}$ factorial of dimension 3.

By induction $R_{N} \cong J_{N-1}\left[\beta_{N}\right] \cong R_{N-1}[X] /\left(\bar{x} X+\bar{\alpha}_{N-1}^{s(N)}-\bar{\alpha}_{N-2}\right)$ and $R_{N-1}$ is a three dimensional domain. $R_{N}$ must be of dimension three, since all the $R_{i}$ are affine rings. Hence $R_{N}$ is factorial of dimension three. 
Remark 1.1. $J_{N}$ is a polynomial ring in three variables. This follows from the fact that $\bar{\psi}$ is an isomorphism (Proposition 1 ).

Corollary 1.1. $x$ is prime in $J$.

Proof. By Proposition 1 and Lemma 1.1, we have $x$ prime in $J_{N}$ for all $N$ since $\phi_{N}(\bar{x})=x$. Thus $x$ is prime in $J=\bigcup J_{N}$.

Corollary 1.2. $J$ is a three dimensional domain.

Proof. Since $J \subseteq k\left(x, y, a_{0}\right)$, it is a domain. Now suppose there exists a chain of prime ideals of length greater than 3 in $J$, say $P_{i} \subsetneq P_{i+1}, i=1,2,3,4$. Since for all $N, J_{N}$ is three dimensional, it must be false that $J_{N} \cap P_{i} \subsetneq P_{i+1} \cap$ $J_{N}$, for all $i$ and all $N$. By the "pigeon-hole" principle, there exists $j$ such that $P_{j} \cap J_{N}=P_{j+1} \cap J_{N}$, infinitely often. This implies that $P_{j}=P_{j+1}$, since $J=$ $\cup J_{K}$ and $J_{K} \subseteq J_{K+1}$, a contradiction.

Therefore $\operatorname{dim} J<4$. That $\operatorname{dim} J=3$ follows from the.proof of the next lemma and the fact that $k\left[x, 1 / x, y / x, a_{0} / x\right]$ is three dimensional.

2. $J$ is factorial.

Lemma 2.1. $J[1 / x]$ is factorial.

Proof. We first calculate $J_{N}[1 / x]$ :

$$
\begin{aligned}
J_{N}[1 / x]= & k\left[x, y, \alpha_{0}, \beta_{1}, \cdots, \beta_{N}\right][1 / x] \\
= & k\left[x, 1 / x, y / x, \alpha_{0} / x,\left((y / x)^{s(2)}-\left(\alpha_{0} / x\right) x\right) \cdot 1 / x,\right. \\
& \left.\quad\left(\left(\left((y / x)^{s(2)}-\left(\alpha_{0} / x\right) x\right) 1 / x\right)^{s(3)}-y / x\right) \cdot 1 / x, \cdots\right] \\
= & k\left[x, 1 / x, y / x, \alpha_{0} / x\right] .
\end{aligned}
$$

Since this is a ring of quotients of a polynomial ring, a factorial ring, it too is factorial. Thus since it is seen that $J_{N}[1 / x]=J_{M}[1 / x]$ for all $N, M, J[1 / x]=$ $J_{N}[1 / x]$ for all $N$. Thus $J[1 / x]$ is a factorial ring.

Remark 2.1. $J[1 / x]$ is Krull. This follows from Lemma 2.1 .

Lemma 2.2. $J=J[1 / x] \cap J(x)$ (We are using Corollary 1.1.)

Proof. Since $J$ is contained in both $J[1 / x]$ and $J(x)$ we will show that $J[1 / x] \cap$ $J_{(x)} \subseteq J$. Let $a \in J[1 / x] \cap J_{(x)}$; write

$$
\alpha=r_{0}+r_{1} / x+\cdots+r_{N} / x^{N}, \quad r_{i} \in J,
$$

as a minimal expression in $N$. Assume $N \geq 1$. Since $a \in J(x)$, there exists $s \in J$ such that $s \alpha \in J$ and $x \nmid s$. Since $s\left(\alpha x^{N-1}-r_{0} x^{N-1}-r_{1} x^{N-2}-\cdots-r_{N-1}\right)=$ $r_{N} \cdot s / x, r_{N} \cdot s / x \in J$. 
Since $x$ is a prime not dividing $s, x$ divides $r_{N}$ in $J$, and the expression for $a$ is not minimal-a contradiction. Hence the lemma.

Lemma 2.3. $J_{(x)}$ is a rank one Dvr.

Proof. The following equivalences hold: $J_{(x)}$ is rank $1 \mathrm{Dvr} \Leftrightarrow J_{(x)}$ is $1-\mathrm{dim}$ $\Leftrightarrow \bigcap(x)^{i}=0$.

Now we must show that $\bigcap(x)^{i}=0$; this will be done in two parts:

Part 1. Let $0 \neq \alpha \in J$. Then there exists $N$ such that $\alpha \in J_{N}$ and $\alpha=$ $\Sigma_{c}{ }_{i j k} x^{i} \beta_{N}^{j} \beta_{N-1}^{k}$. Since $J_{n}$ is the polynomial ring $k\left[x, \beta_{n}, \beta_{n-1}\right]$, by Remark 1.1, for $\alpha^{\prime} \in J_{m}$ :

$\operatorname{deg}_{x}^{(m)} \alpha^{\prime}, \operatorname{deg}_{\beta_{K}}^{(m)} a^{\prime}, K=m, m-1$, are well defined

where $\operatorname{deg}_{x}^{(m)} \alpha^{\prime}$ is the degree of $\alpha^{\prime}$ considered as

a polynomial in the variable $x$ in $J_{m}$; and

$\operatorname{deg}_{\beta_{K}}^{(m)} \alpha^{\prime}$ is the degree of $\alpha^{\prime}$ considered as a poly-

nomial in the variable $\beta_{K}$ in $J_{m}, K=m, m-1$.

We shall show in this part that if $s(N+1)>\operatorname{deg}_{\beta_{N}}^{(N)} \alpha$, then

$$
x^{\left(\operatorname{deg}_{x}^{(N)} a\right)+1}
$$

does not divide $\alpha$ in $J_{m}$, for all $m \geq N$. Now let $t_{1}=\operatorname{deg}_{\beta_{N}}^{(N)} \alpha, t_{2}=\operatorname{deg}_{\beta_{N-1}^{(N)}}^{(N)} a$, $t_{3}=\operatorname{deg}_{x}^{(N)} \alpha$, and assume $s(N+1)>t_{1}$. Simply, we must show that $\alpha$ expressed as a polynomial in $J_{m}, m \geq N$, has a nonzero term $d_{m} x^{a}{ }_{m} \beta_{m}^{b}{ }_{m} \beta_{m-1}^{c}$ where $d_{m} \neq 0 \in k$ and $a_{m} \leq t_{3}$-i.e., the sub $\operatorname{deg}_{x}^{(m)} \alpha$ is less than or equal to $t_{3}$.

Clearly $x^{t 3+1}$ does not divide $\alpha$ in $J_{N}$. We have $\alpha$ as it appears in $J_{N+1}$ :

$$
\begin{aligned}
\alpha & =\sum c_{i j k} x^{i} \beta_{N}^{j}\left(x \beta_{N+1}+\beta_{N}^{s(N+1)}\right)^{k} \\
& =\sum_{0 \leq w \leq k ; i, j, k} c_{i j k} x^{i+w} \beta_{N+1}^{w} \beta_{N}^{j+s(N+1)(k-w)} d_{i j k w}
\end{aligned}
$$

where $0 \neq d_{i j k w} \in k$ since $k$ is of characteristic 0 . There exist $i_{0}, j_{0}$ such that $c_{i_{0, j 0, t_{2}}} \neq 0$ and $j_{0} \geq \max \left\{j \mid \exists i\right.$ s.t. $\left.c_{i j t_{2}} \neq 0\right\}$. It is easy to see that $\left(j_{0}, t_{2}\right)$ is the unique element of the set $\left\{(j, k) \mid \exists i\right.$ s.t. $c_{i j k} \neq 0$ and $j+s(N+1) k$ is maximum $\}$, because $s(N+1)>t_{1}$. Now if we express $\alpha$ as a polynomial in $\beta_{N}$ in $J_{N+1}$, it will look like

$$
a=\beta_{N}^{j_{0}+s(N+1) t_{2}} \cdot b_{0}+f_{0}, \quad 0 \neq b_{0}, f_{0} \in J_{N+1},
$$

where $\operatorname{deg}_{\beta_{N}}^{(N+1)} f_{0}<j_{0}+s(N+1) t_{2}$ and $x^{t_{3}+1} \nmid b_{0}$ in $J_{N+1}$. Therefore $x^{t 3+1}+\alpha$ in $J_{N+1}$. 
We now write $\alpha$ as it appears in $J_{N+2}$ :

$$
\begin{array}{r}
\sum_{\substack{0 \leq w \leq k \\
0 \leq v \leq j+s(N+1)(k-w)}} c_{i j k} x^{x^{i+w+v}} \cdot \beta_{N+2}^{v} \cdot \beta_{N+1}^{w+s(N+2)(j+s(N+1)(k-w)-v)} \cdot d_{i j k w \nu}, \\
\text { where } 0 \neq d_{i j k w v} \in k .
\end{array}
$$

Clearly,

$$
\stackrel{(N+2)}{\operatorname{deg}} \beta_{N+1} \alpha=s(N+2)\left(j_{0}+s(N+1) t_{2}\right)
$$

and

$$
\alpha=\beta_{N+1}^{s(N+2)\left(j_{0}+s(N+1) t_{2}\right)} \circ b_{1}+f_{1}, \quad 0 \neq b_{1}, f_{1} \in J_{N+2},
$$

where

$$
\stackrel{(N+2)}{\operatorname{deg}} \beta_{N+1} f_{1}<s(N+2)\left(j_{0}+s(N+1) t_{2}\right)
$$

and

$$
x^{t_{3}+1}+b_{1} \text { in } J_{N+2} \text {. }
$$

Therefore $x^{t^{3+1}}+\alpha$ in $J_{N+2}$.

In general, we see that, in $J_{N+r}$,

$$
\begin{gathered}
\quad \sum_{0 \leq w_{i} \leq s_{i} ; s, \geq 0} c_{i j k} \\
(*) \quad w_{r} \leq g_{r-1}\left(w_{1}, \cdots, w_{r-1}, s(N+1), \cdots, s(N+r-1), j, k\right) \\
\\
\quad x^{i+\Sigma_{\delta=1}^{r} w_{\delta}} \cdot \beta_{N_{+r}}^{w_{r}} \cdot \beta_{N+r-1}^{g_{r}\left(w_{1}, \cdots, w_{r}, s(N+1), \cdots, s(N+r), j, k\right)} \cdot d_{i j k, w_{1}, \cdots, w_{r}} ;
\end{gathered}
$$

(a) where for fixed $j, k, g_{r}(0, \cdots, 0, s(N+1), \cdots, s(N+r), j, k)>$ $g_{r}\left(a_{1}, \cdots, a_{r}, s(N+1), \cdots, s(N+r), j, k\right)$ if there exists $q$ such that $a_{q}>0$, and (b)

$$
\begin{aligned}
& g_{r}\left(0, \cdots, 0, s(N+1), \cdots, s(N+r), j_{0}, t_{2}\right) \\
& >\max \left\{g_{r}(0, \cdots, 0, s(N+1), \cdots, s(N+r), j, k)\right. \\
& \text { s.t. either } \left.k \neq t_{2} \text { or } j \neq j_{0} \text {, and } \exists i \text { s.t. } c_{i j k} \neq 0\right\}
\end{aligned}
$$

since $s(N+1)>t_{1}$. Putting (a) and (b) together we see that

$$
\alpha=\beta_{N+r-1}^{P(r)} \cdot b_{r-1}+f_{r-1}, \quad 0 \neq b_{r-1}, \quad f_{r-1} \in J_{N+r},
$$

where

$$
\begin{aligned}
& \stackrel{(N+r)}{\operatorname{deg}} \beta_{N+r-1} \alpha=g_{r}\left(0, \ldots, 0, s(N+1), \ldots, s(N+r), j_{0}, t_{2}\right)=P(r), \\
& \quad \begin{array}{l}
(N+r) \\
\operatorname{deg} \beta_{N+r-1}
\end{array} f_{r-1}<P(r), \text { and } x^{t^{3}+1} \nmid b_{r-1} \text { in } J_{N+r^{\circ}}
\end{aligned}
$$


Thus $x^{t^{3+1}} \nmid \alpha$ in $J_{N+r}$ for all $r$.

This completes Part 1 of Lemma 2.3.

Remark 2.2. A bound on the value of $g_{r}$ can now be established: $s(N+r) \cdots$ $s(N+2)\left(t_{1}+s(N+1) t_{2}\right)$; this can be seen by using (a) and (b) in Part 1 , above.

Part 2. Let $0 \neq \alpha \in J$ such that $\alpha \in J_{N}$, and write

$$
\alpha=\sum c_{i j k} x^{i} \beta_{N}^{j} \beta_{N-1}^{k} \text {. }
$$

We shall show that there exists $m$ such that $s(m)>\operatorname{deg} \beta_{m-1}^{(m-1)} \alpha$. Let $r>0$. We will rewrite $\alpha$ in terms of $\beta_{N+r}, \beta_{N+r-1}$, and $x$ in $J_{N+r^{\circ}}$. We note that a bound for $\operatorname{deg}{ }_{\left.\beta_{N+r}+r\right)}^{(N+1)}$ is bounded by a bound for $g_{r-1}\left(u_{1}, \cdots, w_{r-1}, s(N+1), \cdots\right.$, $s(N+r-1), j, k)$. This follows from (*) of Part 1. By Remark 2.2, we see that such a bound is

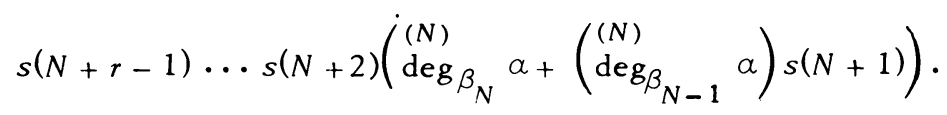

So if we let $m=\operatorname{deg}_{\beta_{N}}^{(N)} \alpha+\left(\operatorname{deg}_{\beta_{N-1}}^{(N)} \alpha\right) s(N+1)+1$ and let

$$
r=\sup \left\{\begin{array}{l}
m-N-1 \\
0
\end{array}\right.
$$

in the above, then $s(N+1)>\operatorname{deg}{ }_{\beta_{N}}^{(N)} \alpha$, if $r=0$ as $s(q)>q$ for all $q$, or $s(m)=$ $s(N+r+1)>\operatorname{deg}{ }_{\left.\beta_{N+r}+r\right)}^{(N+2} \alpha=\operatorname{deg}{ }_{\beta_{m-1}}^{(m-1)} \alpha$ if $r \neq 0$.

This completes Part 2 of Lemma 2.3.

Putting both parts together, we see that if $0 \neq \alpha \in J$, there exists $C$ such that $x^{C} \nmid \alpha$ in $J_{N}$ for all $N$. Thus $\alpha \notin \bigcap(x)^{i}$ since $J=\bigcup J_{P}, J_{P} \subseteq J_{P+1}$, and therefore $\bigcap(x)^{i}=0$. The proof of Lemma 2.3 is complete.

Proposition 2.1. $J$ is a Krull ring.

Proof. The proposition follows from Lemmas 2.2, 2.3 and Remark 2.1.

Proposition 2.2. $J$ is factorial.

Proof. By Corollary 1.1, $S=\left\{x^{K} \mid K \geq 0\right\}$ is a multiplicative system generated by a prime element. From Lemma 2.1 we have $S^{-1} J$ factorial. So by Proposition 2.1 and Theorem $1, J$ is factorial.

\section{3. $J$ is non-Noetherian.}

Lemma 3.1. Let $n \geq 1$. Then $x+\beta_{n}$ in $J$.

Proof. Suppose so. Since $\beta_{n}-x \beta_{n+2}=\beta_{n+1}^{s(n+2)}$, by Corollary $1.1, x \mid \beta_{n+1}$ in $J$. Since $\beta_{n+p}-x \beta_{n+p+2}=\beta_{n+p+1}^{s(n+p+1)}, p \geq 0$, we may deduce in a similar fashion

$$
x \mid \beta_{m} \text { in } J \text { for } m \geq n
$$


We claim this implies $x^{p} \mid \beta_{n}$ for all $p$ in $J$, which is impossible in view of Lemma 2.3 .

Let $p>0$.

$$
\begin{aligned}
\beta_{n}= & x \beta_{n+2}+\beta_{n+1}^{s(n+2)} \\
= & x\left(x \beta_{n+4}+\beta_{n+3}^{s(n+4)}\right)+\left(x \beta_{n+3}+\beta_{n+2}^{s(n+3)}\right)^{s(n+2)} \\
= & x^{2}\left(x \beta_{n+6}+\beta_{n+5}^{s(n+6))}+x\left(x \beta_{n+5}+\beta_{n+4}^{s(n+5)}\right)^{s(n+4)}\right. \\
& +\left(x\left(x \beta_{n+5}+\beta_{n+4}^{s(n+5)}\right)+\left(x \beta_{n+4}+\beta_{n+3}^{s(n+4)}\right)^{s(n+3)}\right)^{s(n+2)} \ldots
\end{aligned}
$$

In view of (1), this implies the claim.

Remark 3.1. Let $s \in J$. We define the constant term of $s$ to be that its representation in any $J_{n}$ it belongs to. This is well defined in view of the particular embedding of $J_{n}$ in $J_{n+1}$.

Lemma 3.2. Let $s \in J$ with nonzero constant term. Let $n>0$. Then

$$
s \beta_{n+1} \notin\left(\beta_{n}, \beta_{n-1}, x\right) J_{r}, \quad r \geq n+1 .
$$

Proof. Induction on $r$ : Since $\left(\beta_{n}, \beta_{n-1}, x\right) J_{n+1}=\left(\beta_{n}, x\right) J_{n+1}$, and $J_{n+1}$ is a polynomial ring, the statement easily follows for $r=n+1$.

Since $\left(\beta_{n}, x\right) J_{n+2}=\left(\beta_{n+1}^{s(n+2)}, x\right) J_{n+2}$, and $J_{n+2}$ is a polynomial ring, the statement again follows for $r=n+2$.

Suppose there exists $r>n+2$ such that $s \beta_{n+1} \in\left(\beta_{n+1}^{s(n+2)}, x\right) J_{r}$. Then there exist $b, c \in J_{r}$ such that $s \beta_{n+1}+b \beta_{n+1}^{s(n+2)}=c x$. Thus $\beta_{n+1}\left(s+b \beta_{n+1}^{s(n+2)-1}\right)=c x$. Since $x$ is prime by Corollary 1.1 , by Lemma 3.1 it must divide $s+b \beta_{n+1}^{s(n+2)-1}$. Since $J_{r}$ is a polynomial ring in the variables $x, \beta_{r-1}, \beta_{r}$, and $\beta_{n+1}$ is a function of those variables without constant term, this is impossible.

This concludes Lemma 3.2.

Proposition 3.1. Let $S$ be a multiplicative system in $J$ such that $S \cap M=\varnothing$ where $M=\left(x, y, \alpha_{0}, \beta_{1}, \cdots, \beta_{k}, \cdots\right) J$. Then $S^{-1} J$ is non-Noetherian.

Proof. Claim: $S^{-1} M$ is not finitely generated. Suppose it were. Then there exists $n$ such that a set of generators of $S^{-1} M$ lie in $J_{n}$. Since $M \cap J_{n} \subseteq$ $\left(x, \beta_{n}, \beta_{n-1}\right) S^{-1} J$, it follows that $S^{-1} M=\left(x, \beta_{n}, \beta_{n-1}\right) S^{-1} J$. This says that there exists $s \in S$ such that $s \beta_{n+1} \in\left(x, \beta_{n}, \beta_{n-1}\right) J$. Since $S \cap M=\varnothing$, and $J=\bigcup J_{m}$, $J_{m} \subset J_{m+1}$, in view of Lemma 3.2 we have a contradiction.

This completes Proposition 3.1 . 


\section{Conclusions.}

Theorem 3. $J$ is a three dimensional non-Noetherian factorial ring, in characteristic zero.

Proof. This follows from Propositions 2.2 and 3.1 and Corollary 1.2.

Corollary 4.1. There exists a three dimensional non-Noetherian quasi-local factorial ring in characteristic zero.

Proof. Let $P=\left(\alpha_{0}, \beta_{1}, \cdots, \beta_{k}, \cdots\right) J$. Since $J / P \simeq k[x]$ a domain, $P$ is prime. By the arguments of Proposition 3.1, $P$ cannot be finitely generated, much less be principal. Since $P \subsetneq M$, we deduce that $M$ has height three. In view of Theorem 3, and Proposition 3.1, $(J \backslash M)^{-1} J$ is our example.

\section{REFERENCES}

1. Pierre Samuel, Lectures on unique factorization domains, Lectures on Math., no. 30, Tata Institute of Fundamental Research, Bombay, India, 1964. MR 35 \#5428.

2. Ibid.

DEPARTMENT OF MATHEMATICS, THE UNIVERSITY OF ROCHESTER, ROCHESTER, NEW YORK 14627

Current address: P. O. Box 43, Tuxedo Park, New York 10987 